\title{
Identification of Recommended Hevea brasiliensis (Willd. ex A. Juss.) Müll. Arg. Clones Grown in Sri Lanka by RAPD Analysis
} \author{
B.W.A.N. Baddewithana ${ }^{3}$ \\ Postgraduate Institute of Agriculture \\ University of Peradeniya \\ Sri Lanka
}

K.K. Liyanage*, V.A. Sumanasinghe ${ }^{1}$, D.P.S.T.G. Attanayake ${ }^{2}$ and

\begin{abstract}
Systematic characterization of rubber (Hevea brasiliensis (Willd. ex A.Juss.) Müll.Arg.) clones based on their molecular diversity, and identification of molecular markers useful for characterization of rubber clones are vital in crop management systems and research. This study was undertaken to estimate the molecular diversity present among the Sri Lankan rubber clones and to determine the importance of molecular markers in categorizing different clones into distinct groups. Thirty eight rubber clones were screened using pre-selected 27 RAPD primers out of which five primers namely, OPC 04, OPA 18, $O P B 18, O P A 20$ and OPS 02 that produced more than three discrete bands on an average were used to assess the genetic diversity of rubber clones. These five primers generated high level of polymorphism among the clones. The number of alleles detected by different primers ranged from 1 to 8 with an average of 6 alleles per primer and the level of polymorphism was $100 \%$. The genetic distance values based on RAPD analysis ranged between 0.157 and 1.000. The resultant Nei's distance matrices were used to create clusters with UPGMA clustering method. Out of the 38 Hevea clones, three clones namely, PB 217, RRISL 201 and RRISL 226 could not be included due to lack of bands (less than three). The remaining 35 clones were grouped into seven main clusters at nearly $85 \%$ distance level. The cluster size ranged from 1 to 16. The RAPD primers OPB 18 and OPS 02 were identified as suitable primers for genetic differentiation of the recommended $\underline{H}$. brasiliensis clones. A key was constructed to identify the 35 clones using RAPD assay.
\end{abstract}

Keywords: Characterization, clones, RAPD profiles, Hevea brasiliensis

\section{INTRODUCTION}

Use of genetically improved high yielding planting materials of rubber in Sri Lanka has achieved spectacular growth in the area, production and most notably the higher productivity during the past few years. (Anon, 2010). These improvements are achieved pursuant to a well conceived research and development program, notably a systematic crop improvement program coupled with an efficient extension and research activities. Rapid adoption of indigenously bred new clones has contributed substantially to attain higher yield both in

1 Department of Agricultural Biology, Faculty of Agriculture, University of Peradeniya. Sri Lanka

2 Faculty of Agriculture and Plantation Management, Wayamba University of Sri Lanka, Makandura, Gonawila, Sri Lanka

3 Genetics and Plant Breeding Department, Rubber Research Institute of Sri Lanka, Nivitigalakele, Matugama, Sri Lanka

Corresponding author: 1kapila@ymail.com 
estate and small holding sector in the country. Systematic breeding program followed by vegetative propagation has resulted in substantial enhancement in productivity of Hevea during the past decades and genetic improvement in Hevea has been described as one of the outstanding success stories in agriculture of $20^{\text {th }}$ century (Attanayake, 2001). From the original "Wickham gene pool" with a productivity ranging from $50-300 \mathrm{~kg} / \mathrm{ha} / \mathrm{yr}$, breeders have developed clones with a production potential exceeding $3000 \mathrm{~kg} / \mathrm{ha} / \mathrm{yr}$. At present, around 50 high yielding clones are available for commercial planting.

However, the genetic base of the existing population is very narrow as rubber breeding in Sri Lanka was largely based on a small population of about 1919 seedlings introduced in 1876 from Wickham collection. In addition, directional selection for a few economically important characteristics such as yield, vigour and disease tolerance and extensive use of (clonal) vegetative propagation have led to further erosion of genetic variability. Consequently, the problems related to breeding and selection of Hevea, such as inbreeding depression which leads to declining yield response have become more apparent. Therefore, in general, present breeding population of rubber does not exhibit highly conspicuous and very distinct variations in its characters (Attanayaka et al., 2000) making it difficult to identify clones at morphological level accurately.

Proper identification of these clones plays a vital role in crop management systems and research. However, the traditional approach of clone identification based on morphological differences poses several limitations, as most of the morphological characters are influenced by the environmental conditions and the age of plant, and they are not variable enough to adequately characterize genetic differences among elite genotypes. Therefore, additional identification parameters are required for both immature budded plants and for bud wood nurseries. The application of molecular strategies in genotype identification is now a common practice. Molecular markers play an important role in this respect, and it can clearly differentiate the genetic material avoiding any of the environmental influences, which cause variations in gene expression (Korakot et al., 2008).

Both hybridization based and PCR based molecular markers have been developed for Hevea (Hermandez et al., 2006 ; Korakot et al., 2008). The DNA based marker procedure leads to a greater understanding of clones or cultivars and these techniques are used by the plant breeders to identify genetic variability among the species and clones or cultivars. Among these, the RAPDs are relatively inexpensive, fast, reliable and appropriate to use in an average molecular laboratory. Although RAPDs are dominant markers and display the limitation in lack of repeatability on certain cases, they do not require any prior sequence information. However, it will provide useful information in understanding the genome differences and genetic relationships among the Hevea clones. Therefore, the objective of the present study was to analyze the genetic diversity of recommended Hevea brasiliensis clones and identify and characterize them by using molecular markers.

\section{MATERIALS AND METHODS}

A total of 38 clones representing 100 series, 200 series, 2000 series and some foreign clones (Table 1) recommended by the Rubber Research Institute of Sri Lanka (Attanayake, 2001) were selected. 
Table 1. Recommended clones and their parent

\begin{tabular}{|l|l|l|}
\hline & Clone & Parentage \\
\hline 01 & RRIC 100 & RRIC 52 x PB 86 \\
\hline 02 & RRIC 102 & RRIC 52 x RRIC 7 \\
\hline 03 & RRIC 121 & PB 28/59 x IAN 45/873 \\
\hline 04 & RRIC 130 & IAN 45/710 x RRIC 45 \\
\hline 05 & RRIC 133 & IAN 45/710 x RRIC 52 \\
\hline 06 & RRISL 201 & RRIC 103 x HP 8501 \\
\hline 07 & RRISL 203 & RRIC 100 x RRIC 101 \\
\hline 08 & RRISL 205 & 82 HP x 82 HP \\
\hline 09 & RRISL 206 & 82 HP x RRIC 101 \\
\hline 10 & RRISL 208 & RRIC 101 x RRIM 600 \\
\hline 11 & RRISL 210 & RRIC 101 x RRIM 600 \\
\hline 12 & RRISL 211 & RRIC 101 x RRIM 600 \\
\hline 13 & RRISL 215 & Illegitimate \\
\hline 14 & RRISL 216 & Illegitimate \\
\hline 15 & RRISL 217 & PB 28/59 x RRIC 121 \\
\hline 16 & RRISL 218 & PB 28/59 x RRIC 121 \\
\hline 17 & RRISL 219 & PB 28/59 x RRIC 102 \\
\hline 18 & RRISL 220 & PB 28/59 x RRIC 121 \\
\hline 19 & RRISL 221 & RRIC 52 x PB 28/59 \\
\hline
\end{tabular}

\begin{tabular}{|l|l|l|}
\hline & Clone & Parentage \\
\hline 20 & RRISL 222 & RRIC 102 x IAN 45/710 \\
\hline 21 & RRISL 223 & PB 28/59 x IAN 45/710 \\
\hline 22 & RRISL 225 & RRIC $102 \times$ PB 28/59 \\
\hline 23 & RRISL 226 & RRIC 102 x PB 28/59 \\
\hline 24 & RRISL 2000 & RRIC 100 x RRIC 101 \\
\hline 25 & RRISL 2001 & RRIC 100 x RRIC 101 \\
\hline 26 & RRISL 2002 & RRIC 100 x RRIC 101 \\
\hline 27 & RRISL 2003 & 82 HP x RRIC 101 \\
\hline 28 & RRISL 2004 & 82 HP x PB 86 \\
\hline 29 & RRISL 2005 & PB 28/59 x IAN 45/710 \\
\hline 30 & RRISL 2006 & PB 28/59 x RIC 36 \\
\hline 31 & PB 86 & Primary clone \\
\hline 32 & PB 28/59 & Primary clone \\
\hline 33 & PB 217 & PB 5/51 x PB 6/9 \\
\hline 34 & PB 235 & PB 5/51 x PB S/78 \\
\hline 35 & PB 255 & PB 5/51 x PB 32/36 \\
\hline 36 & PB 260 & PB 5/51 x PB 49 \\
\hline 37 & PR 305 & Tjir $1 \times$ BD 2 \\
\hline 38 & RRIM 712 & RRIM 605 x RIM 71 \\
\hline
\end{tabular}

RRIC - Rubber Research Institute of Ceylon

RRISL - Rubber Research Institute of Sri Lanka

RRIM - Rubber Research Institute of Malaysia

PB - Prang Besar, Malaysia

PR - Proefstation voor Rubber, Indonesia

\section{Isolation of DNA}

Immature leaves of the "apple green stage" from each clone were collected. Genomic DNA was extracted from the collected fresh leaves using the procedure described by Withanage \& Attanayake (2005). According to the protocol, nine volumes of extraction buffer [100 mM Tris HCL (pH 8.0), $50 \mathrm{mM}$ EDTA and $500 \mathrm{mM} \mathrm{NaCl}$ ] was mixed with one volume of $10 \%$ SDS (Sodium Dodecyl Sulphate) to prepare the extraction solution.

Twenty $\mathrm{mg}$ of leaf materials were ground quickly with $800 \mu \mathrm{l}$ of extraction solution in a mortar. Then the liquid phase was transferred into a $2 \mathrm{ml}$ eppendorp tube which had been placed in ice. This was mixed well by slowly inverting the tubes with equal volume of chloroform for about 5 minutes and spun at $7500 \mathrm{rpm}$ for 4.5 minutes. After centrifugation, the supernatant was transferred into another tube. Then $700 \mu \mathrm{l}$ of $100 \%$ Ethanol was added. It was then mixed well and centrifuged at $7500 \mathrm{rpm}$ for 4.5 minutes to pellet out DNA. After removing the supernatant, the DNA pellet was washed twice with $100 \mu 1$ of $70 \%$ Ethanol and subsequent centrifugation at $1000 \mathrm{rpm}$ for one minute. Then DNA was dried at room temperature. The DNA pellet was re-suspended in $50 \mu 1$ of autoclaved ultra purified water and stored at $4{ }^{\circ} \mathrm{C}$.

In order to maintain consistency between samples, the purity and the concentration of DNA samples were determined using the spectrophotometer and electrophoresis methods (Fonseka et al., 2005). 


\section{PCR amplification for RAPD assay}

Genomic DNA samples of the 38 clones were amplified using different decamer primers from Operon Technologies, Almeda, USA, and GoTaq DNA polymerase (Promega- USA). Each amplification was performed two to three times in separate experiments to ensure the reproducibility of the results. After $1.4 \%$ agarose gel electrophoresis, the images under UV trans-illuminator were captured and scored visually for presence or absence of bands.

\section{Molecular data analysis}

The amplification profiles were used to estimate genetic similarity among different clones on the basis of shared amplification products. Highly polymorphic primers were used to evaluate the individuals. The RAPD patterns were scored on the basis of presence or absence of bands. The band is an amplification product produced by complete or partial nucleotide sequence homology between the template DNA and a random primer within a certain amplification distance. Amplification of several discrete loci in the genome by a random primer is a useful way to screen polymorphism between individuals. The similarity coefficients were utilized to generate a dendrogram using UPGMA (Unweighted Pair Group Method of Arithmetic means) and RAPDistance 1.31 RAPD data analyzing software package (Nei \& Li, 1979).

\section{RESULTS AND DISCUSSION}

In this RAPD assay, out of the pre-selected 27 primers, five primers OPC 04, OPA 18, OPB 18, OPA 20 and OPS 02 which produced more than three discrete bands on average were used to assess the genetic diversity of Hevea brasiliensis clones. Out of 38 clones, three clones namely PB 217, RRISL 201 and RRISL 226 were opted out due to lack of bands (less than three). These five primers generated high level of polymorphism among the clones selected. The number of alleles detected by different primers ranges from 1 to 8 with an average of 6 alleles per primer and the level of polymorphism was $100 \%$. This indicates that, these primers are useful to determine the genetic differences among the rubber clones and to study their phylogenetic relationship.

There were some studies on Hevea, proving the usefulness of RAPD techniques in finding polymorphism. Attanayake et al. (2000) reported that nine out of sixty primers were highly polymorphic between two clones namely RRIC 102 and GPS II. Fonseka et al. (2005) reported that out of 47 primers, three were highly polymorphic between eleven clones of RRISL 200 and RRISL 2000 series. Hermandez et al. (2006) reported, out of 102 random primers, only 23 showed polymorphism in the study of genetic variation of rubber clones of Asian, South and Central American origin.

RAPD profile of primer OPB 18 (Fig. 1) showed bands for 28 clones tested while 10 clones did not produce any repeatable bands. Out of these 28 clones, 15 clones produced polymorphic bands, which was very useful in clone identification process. 


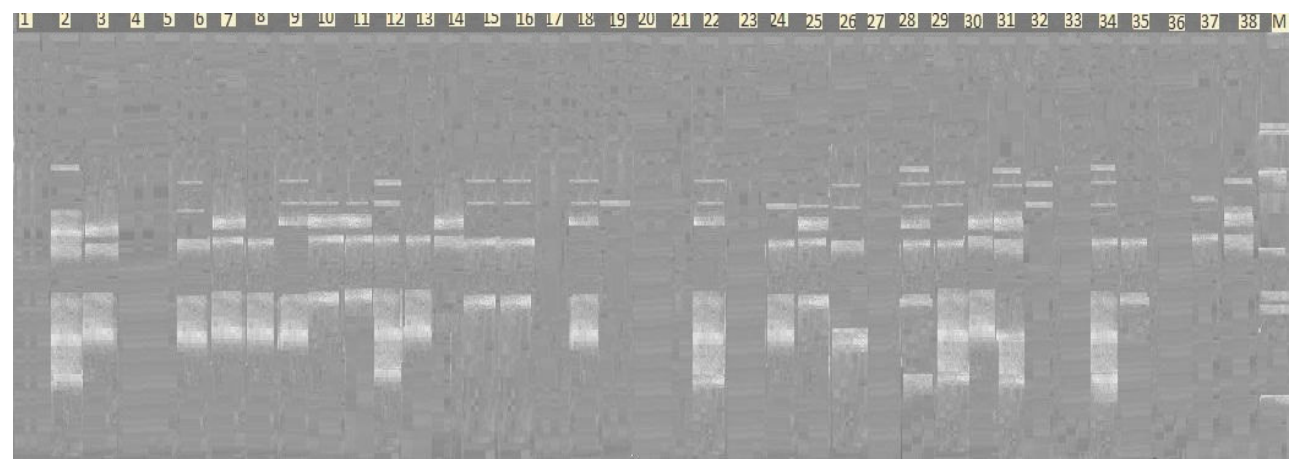

Fig. 1. RAPD profiles for primer OPB 18

Lane M $1 \mathrm{~kb}$ molecular marker

Lane 1 to 38 - RRIC 100, RRIC 102, RRIC 121, RRIC 130, RRIC 133, RRISL 2000, RRISL 2001, RRISL 2002, RRISL 2003, RRISL 2004, RRISL 2005, RRISL 2006, PB 86, PB 217 , PB 235, PB 255, PB 260, PB 28/59, PR 305, RRIM 712, RRISL 201, RRISL 203, RRISL 205, RRISL 206, RRISL 208, RRISL 210, RRISL 211, RRISL 215, RRISL 216, RRISL 217 , RRISL 218, RRISL 219, RRISL 220, RRISL 221, RRISL 222, RRISL 223, RRISL 225, RRISL 226,

RAPD profile generated from primer OPS 02 (Fig. 2) showed bands for 24 clones tested and 14 clones did not produce any repeatable bands. Out of these 24 clones, 14 clones produced polymorphic bands, which was significant in clone identification process.

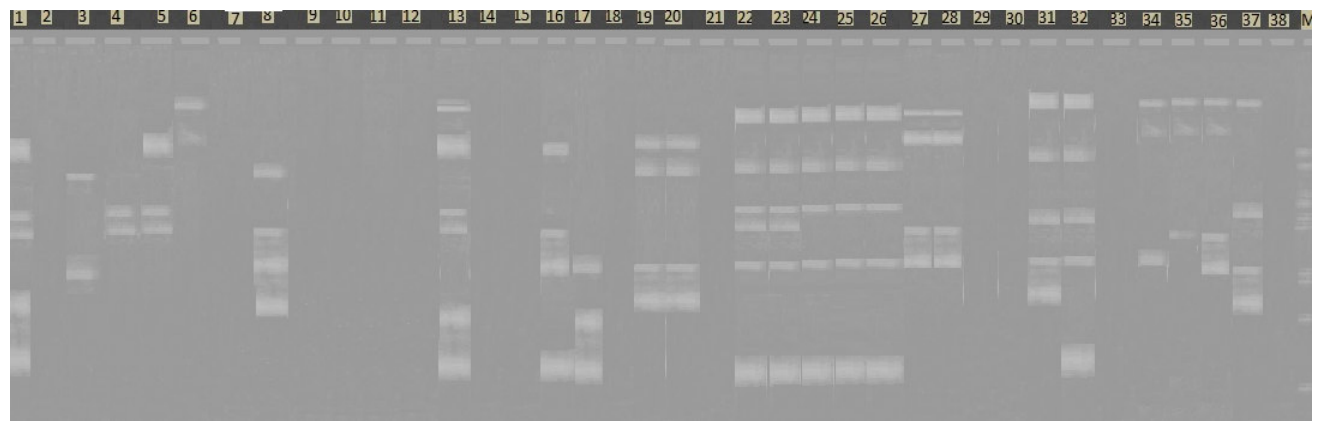

Fig. 2. RAPD profiles for primer OPS 02

Lane M $1 \mathrm{~kb}$ molecular marker

Lane 1 to 38 - Legend as in Fig. 1

Nei's distance matrix was used to calculate the genetic distances between clones. The banding patterns of RAPD scored in the form of binary data were used for computing the genetic distance matrix. These genetic distance values obtained for each pair wise comparison among the 35 clones are presented in the Table 2. The genetic distance values based on RAPD bands ranged from 0.157 to 1.000 . Among the 35 clones under study, the highest genetic distance (1.000) was recorded between set of clone pairs highlighted in the Table 2. The reasons for such higher value for the genetic distances other than the actual polymorphism could be due to the limited number of primers used. 
The lowest genetic distance of 0.153 was recorded between the clones RRISL 206 and RRISL 210 and it indicated that these two clones are genetically the closest amongst the 35 clones studied. The next most similar ones were the RRISL 217 and RRISL 2003 with the value of 0.157 . These results can be used in selecting clones (parents) with high genetic distance for hand pollination programs to obtain higher heterosis effects in the progenies generated.

The resultant Nei's distance matrices were used to create clusters of Hevea brasiliensis clones with UPGMA clustering method. Two clones will be joined together to form a cluster if they are least different from each other in their genetic distances. The 35 Hevea clones tested were grouped into seven main clusters at nearly 85\% distance level (Fig. 3). The cluster size ranged to encompass from one to sixteen clones. The list of all seven clusters along with the clones included is presented in Table 3.

Among these clusters, clusters 3, 4 and 7 contained only single clones RRISL 203, RRISL 219 and PB 255, respectively, indicating their higher genetic diversity to the rest of clones studied.

\section{Clone Identification based on RAPD Profiles}

An effort was made to develop a RAPD based key with a minimum number of primers to identify the 38 clones recommended for planting.

In the first step of the key, RAPD profiles generated from the primer OPC 04 was used. Out of the 38 clones tested, 24 clones did not produce repeatable bands and remaining clones produced five different clusters in the key. If the clusters contain more than one clone, they could be separated by using other primers as shown in Fig. 4.

Then, the set of clones that did not produce repeatable bands with primer OPC 04 were subjected to reaction with OPA 18. Of those 24 clones, 11 clones did not produce repeatable bands and the remaining 13 clones produced five different clusters in the key. If more than one clone is present within any of five sets of clusters, these clones can be separated by using primer OPB 18 and OPB 20.

The 11 clones that did not produce bands with primer OPA 18 could be separated into different clusters when they were subjected to primer OPB 18, OPN18 and OPS 2 (Fig. 4). 
Table 2. Average genetic distance between clones

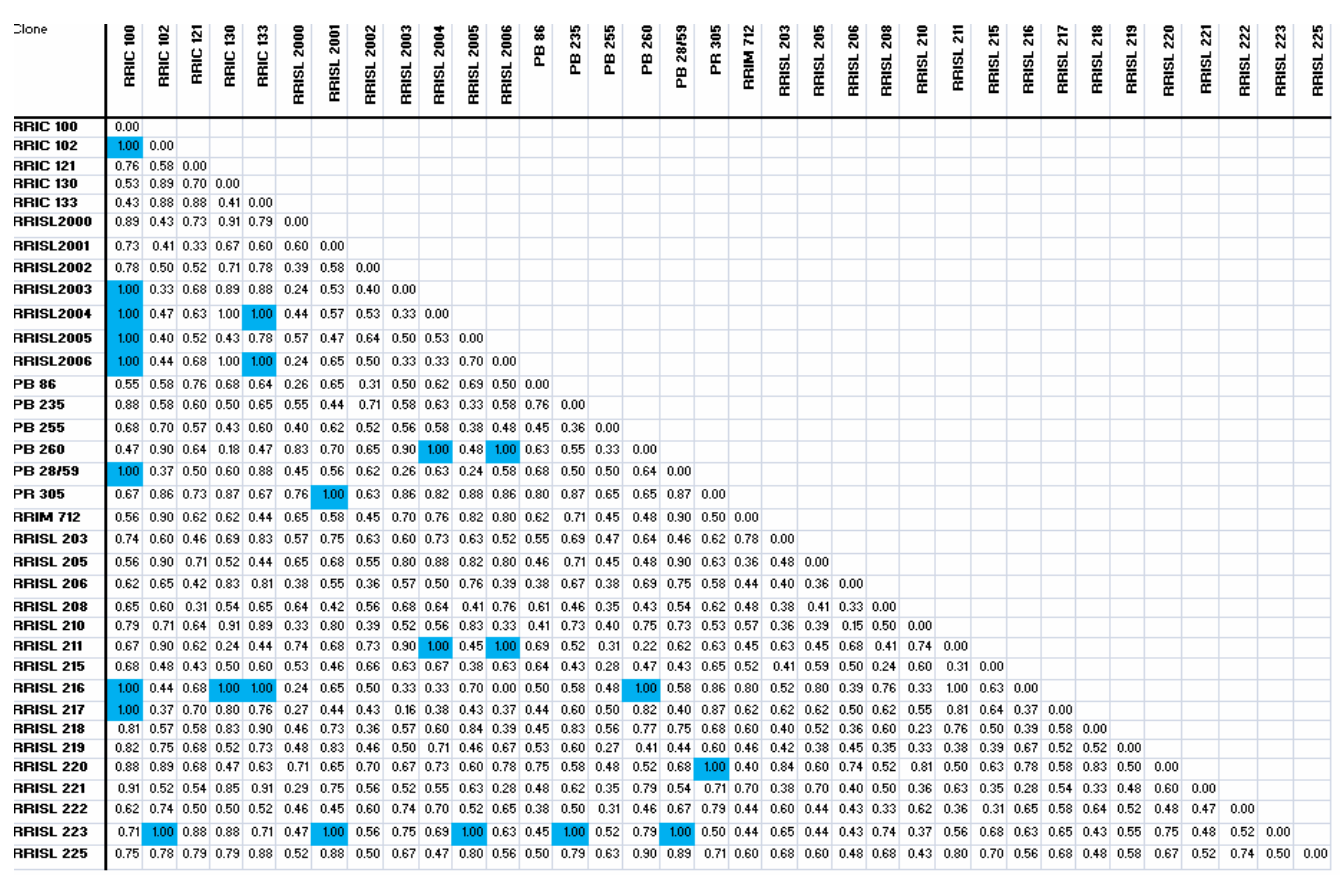

Table 3. The cluster composition of different clones based on the RAPD profiles

\begin{tabular}{|c|c|c|}
\hline $\begin{array}{l}\text { Cluster } \\
\text { No. }\end{array}$ & $\begin{array}{l}\text { Number of } \\
\text { clones }\end{array}$ & Clone name \\
\hline 1 & 16 & $\begin{array}{l}\text { RRIC 102, RRISL 2005, PB 28/59, RRISL 2003, RRISL 217, } \\
\text { RRISL 2004, RRISL 2006, RRISL 216, RRISL 2000, RRISL 221, } \\
\text { RRISL 2002, PB 86, RRISL 225, RRISL 206, RRISL 210, RRISL } \\
218,\end{array}$ \\
\hline 2 & 2 & PR 305, RRISL 223 \\
\hline 3 & 1 & RRISL 203 \\
\hline 4 & 1 & RRISL 219 \\
\hline 5 & 8 & $\begin{array}{l}\text { RRIC 100, RRIC 133, RRIC 130, PB 260, RRISL 211, RRISL } \\
\text { 205, RRIM 712, RRISL } 220\end{array}$ \\
\hline 6 & 6 & $\begin{array}{l}\text { RRISL 215, RRISL 208, RRIC 121, RRISL 2001, PB 235, RRISL } \\
222\end{array}$ \\
\hline 7 & 1 & PB 255 \\
\hline
\end{tabular}




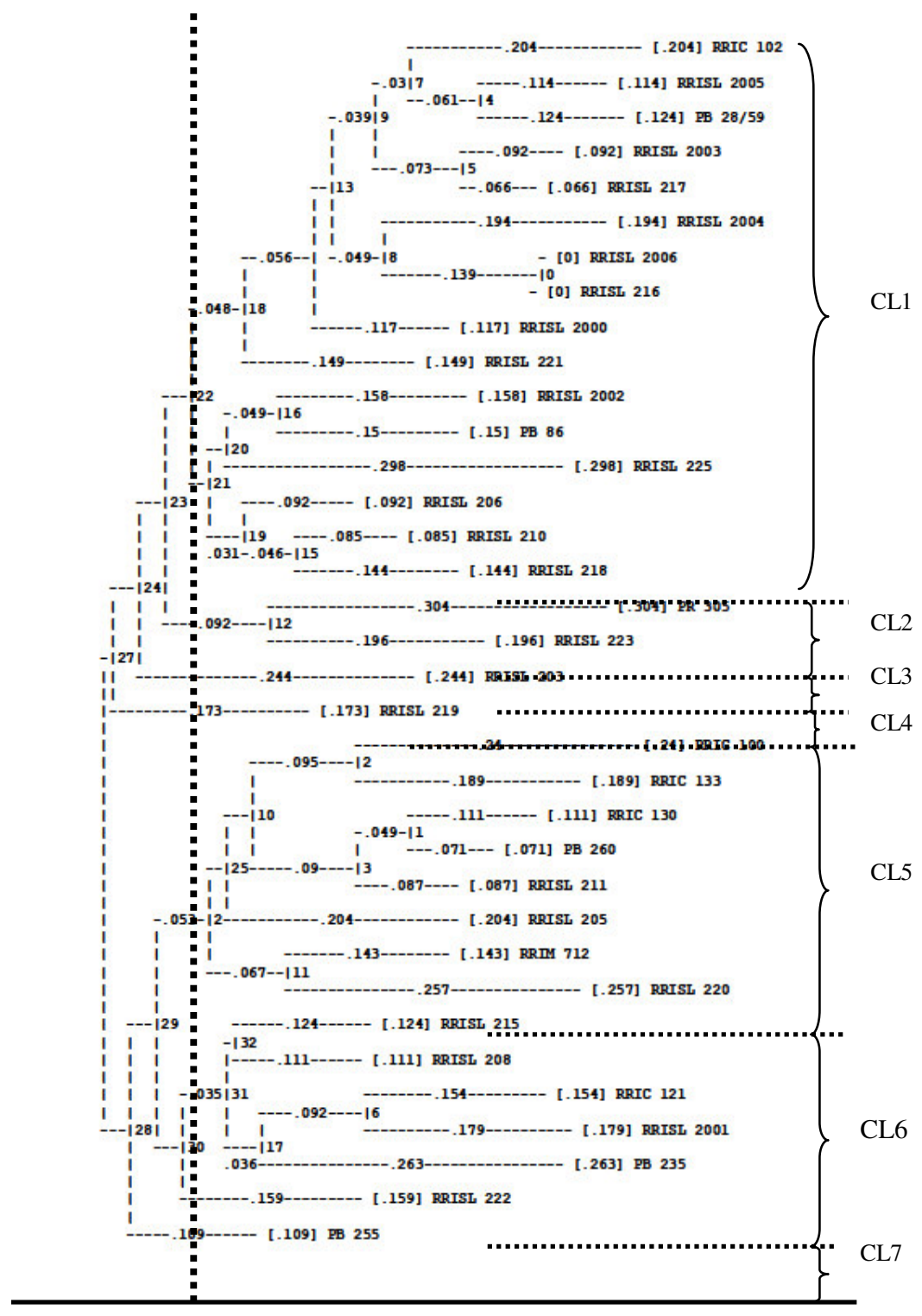

Fig. 3. The cluster diagram showing the genetic relatedness of the 35 Hevea brasiliensis clones

CL 1 to CL 7 are different clusters identified at the $85 \%$ distance level. 


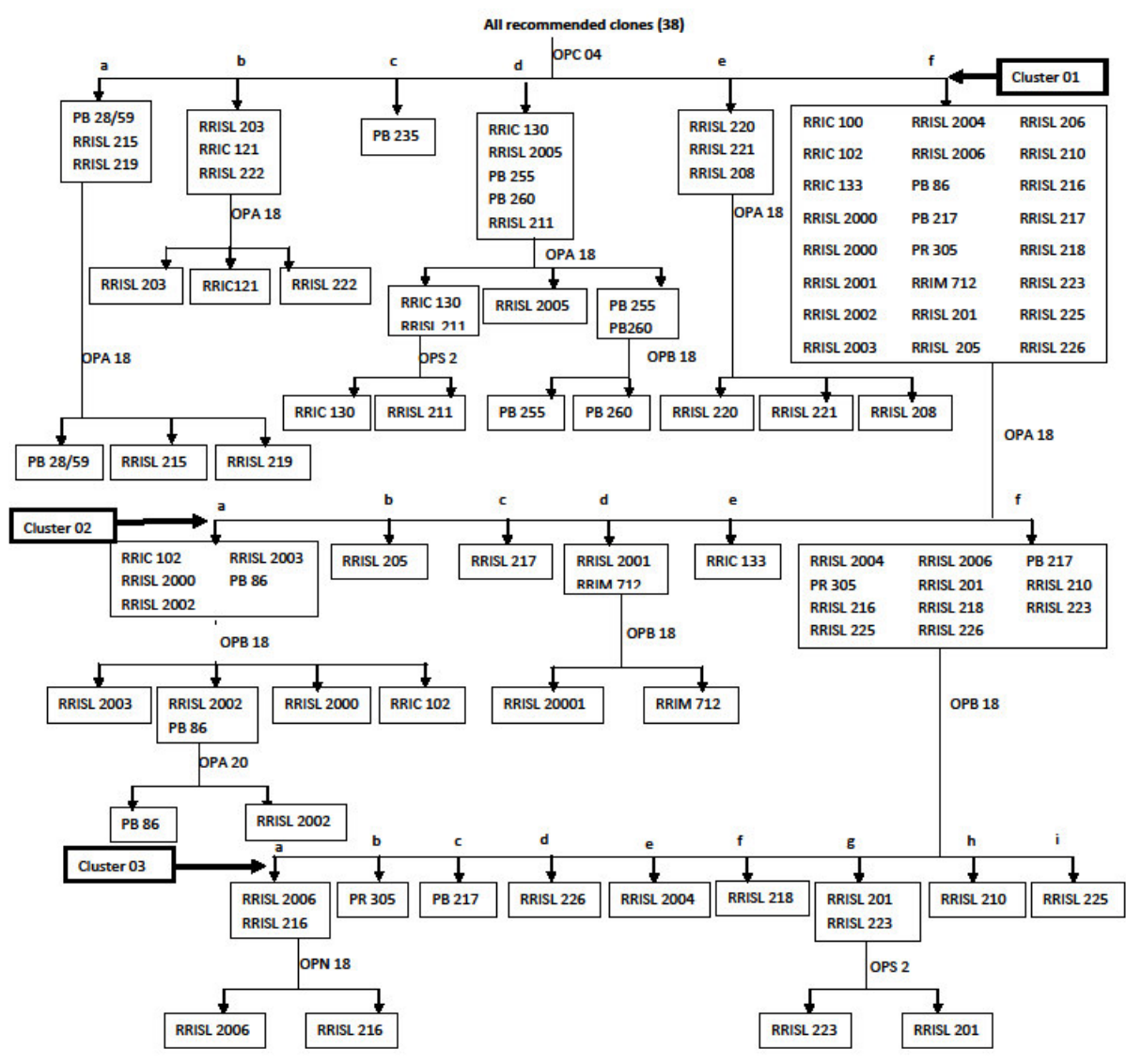

Fig. 4. The clone identification key

In this study, out of 27 primers tested, six primers (OPC 04, OPA 18, OPB 18, OPA 20, OPS 02 and OPN 18) resulted in discrete banding patterns for identification of the entire set of clones used. It could be used to identify different clones from the set of clone mixture as shown in Table 4. If the rubber plantation consists with a mixture of clones having either one clone from each set given under different primer category as shown in Table 4, then these clones could be identified directly using the relevant primer. This is very important in clone identification process because most of the rubber plantations consist of only a few recommended clones and, those can be identified directly deploying just one primer which saves chemicals, money and the time. 
Table 4. Set of clones identified directly by different primers

\section{Primer OPC 04}

Set of Clones

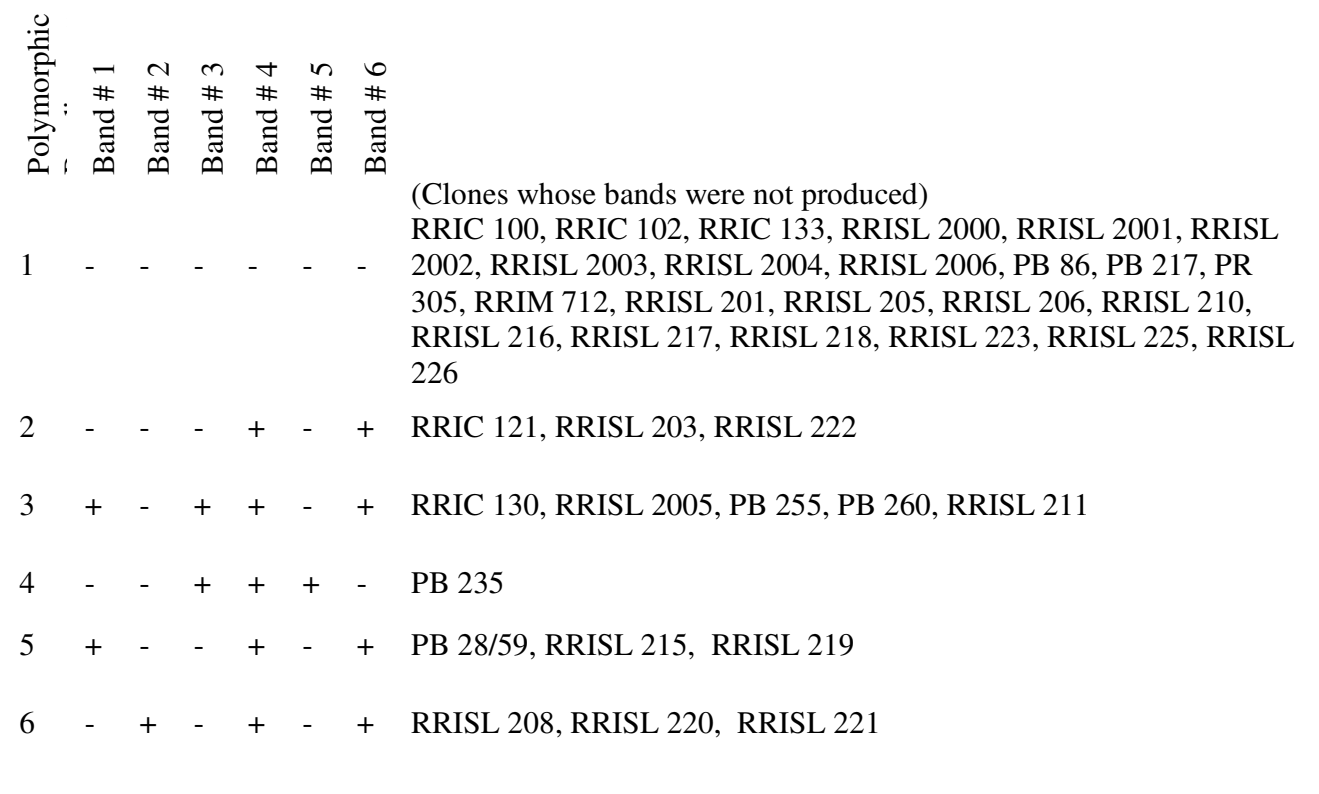

Primer OPA 18

Set of Clones

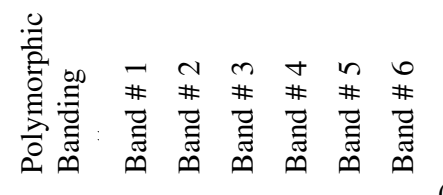

(Clones whose bands were not produced)

RRISL 2004, RRISL 2006, PB 217, PR 305, RRISL 201, RRISL

1 - $\quad$ - $\quad-\quad$ 203, RRISL 210, RRISL 216, RRISL 218, RRISL 221, RRISL 223, RRISL 225, RRISL 226

$2++\quad-\quad-\quad-\quad$ RRIC 100, RRIC 121, RRISL 206

3 - - + - + RRIC 102, RRISL 2000, RRISL 2002, RRISL 2003, PB 86, PB $28 / 59$

$4-+-++-$ RRIC 130, PB 235, PB 255, PB 260, RRISL 205, RRISL 211, RRISL 220

$5 \quad-\quad+\quad+\quad+\quad+$ RRIC 133

$6++-\quad+\quad-\quad$ RRISL 2001, RRIM 712, RRISL 208, RRISL 215, RRISL 222

$-\quad+\quad+\quad+\quad-\quad$ RRISL 2005, RRISL 217 RRISL 219

Table 4 Continued 


\section{Primer OPB 18}

Set of Clones

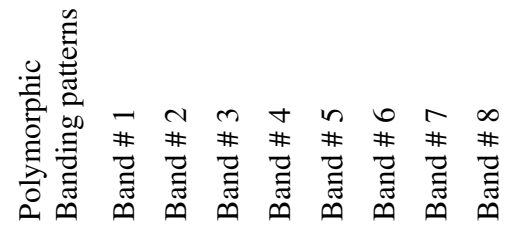

(Clones whose bands were not produced)

RRIC 100, RRIC 130, RRIC 133, PB 260, RRIM 712,

1 RRISL 201, RRISL 205, RRISL 211, RRISL 220, RRISL 223

$2+-++++++$ RRIC 102

3 - $\quad-\quad+\quad+\quad+\quad-$ RRIC 121, RRISL 2001

$4-++-+++-$ RRISL 2000

$5 \quad-\quad-\quad-\quad+\quad+\quad+\quad-\quad$ RRISL 2002, PB 86

$6-+++-+\quad-\quad$ RRISL 2003, PB 28/59

$7 \quad-\quad++++-\quad-\quad$ RRISL 2004, RRISL 2005, RRISL 208

$8-++-++++$ RRISL 2006, RRISL 216

$9 \quad-\quad-\quad+\quad+\quad-\quad-$ PB 217

$10-\quad+\quad+\quad++-\quad-\quad$ PB 235, PB 255

$11-\quad+\quad-\quad-\quad-$ PR 305

$12-+++-+++$ RRISL 203

$13-{ }_{1}+{ }_{-}+++-$RRISL 206

$14-++-+-+-$ RRISL 210

$15++++++-+$ RRISL 215

$16-{ }_{1}++-++$ RRISL 217

$17++-++-++$ RRISL 218

$18-++\quad-\quad-\quad-\quad$ RRISL 219

$19+++-++++$ RRISL 221

$20-$ - $_{2} \quad-\quad+\quad+\quad-\quad$ RRISL 222

$21-\quad-\quad+\quad+\quad+\quad-\quad$ - RRISL 225

$22-\quad+\quad+\quad+-\quad-\quad$ RRISL 226

Table 4 - Continued,,,,,,,

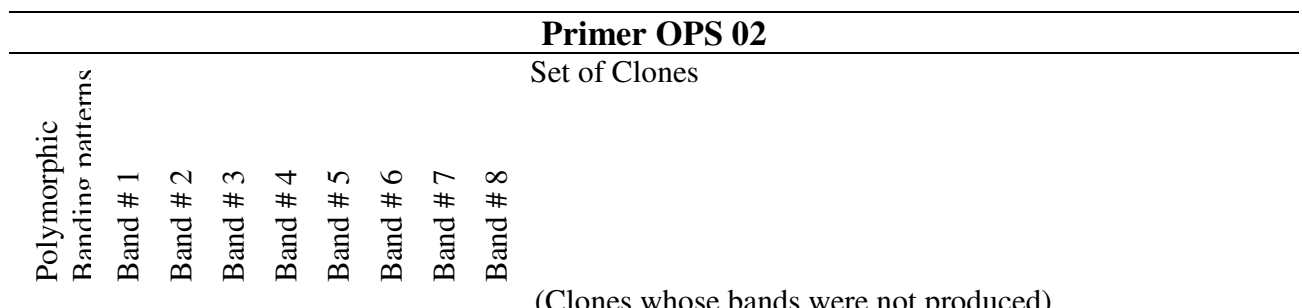

(Clones whose bands were not produced)

RRIC 102, RRISL 2001, RRISL 2003, RRISL 2004, RRISL 2005, RRISL 2006, PB 217, PB 235, PB 28/59,

$\begin{array}{llllllllll}1 & - & - & - & - & - & - & - & - & R R I S L ~ 201, ~ R R \\ & & & & & & & & & 226 \\ 2 & - & + & - & + & + & - & + & + & \text { RRIC 100 } \\ 3 & - & - & + & - & - & + & - & - & R R I C ~ 121 \\ 4 & - & - & - & + & + & - & + & - & \text { RRIC 130 } \\ 5 & - & + & - & + & + & - & - & - & \text { RRIC 133 } \\ 6 & + & + & - & - & - & - & - & - & R R I S L ~ 2000 \\ 7 & - & - & + & - & + & + & + & - & \text { RRISL 2002 }\end{array}$




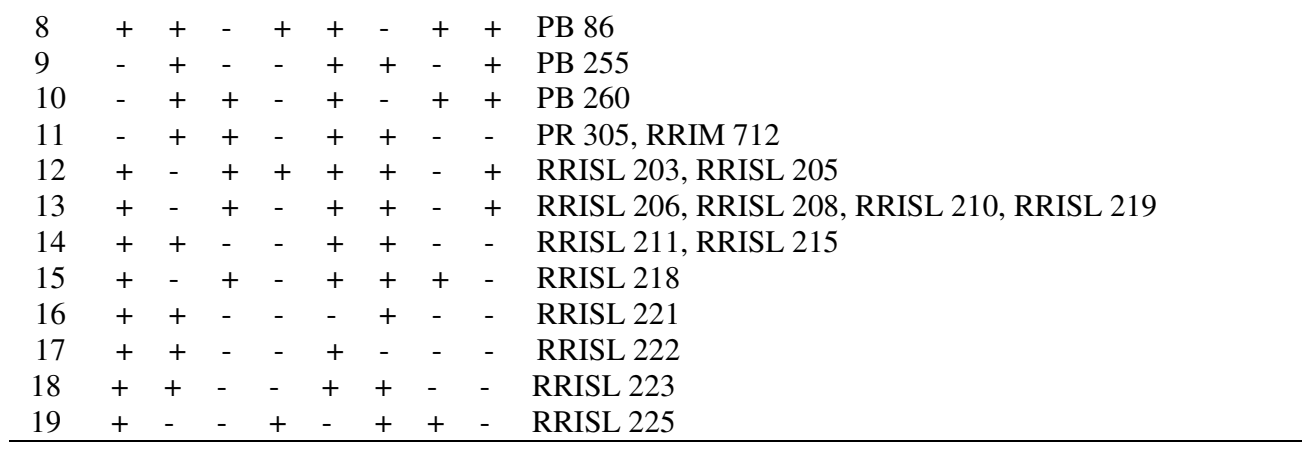

Table 4 - Continued

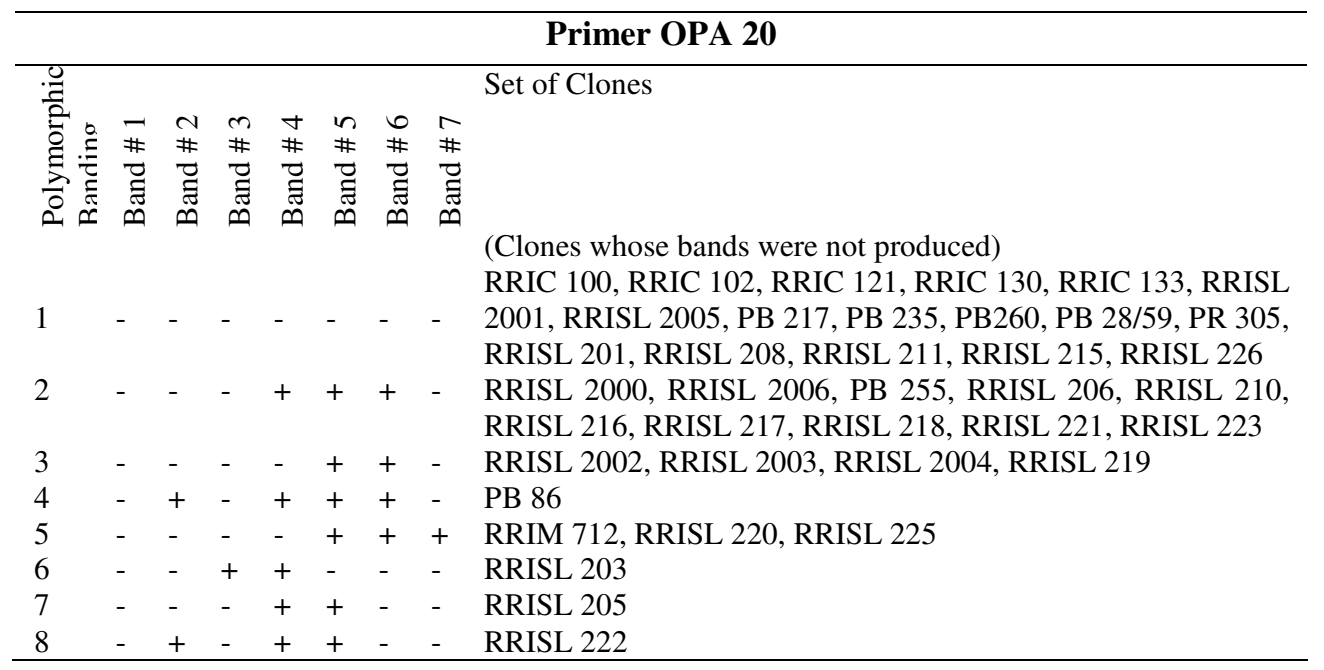

Similar results were obtained by Fonseka et al. (2005) in identifying eleven RRISL 200 series and RRISL 2000 series clones. Of the 11 clones, 7 clones (RRISL 216, RRISL 218, RRISL 220, RRISL 223, RRISL 2000, RRISL 2003 and RRISL 2006) could be separated out by primer OPA 20 and remaining 4 clones (RRISL 210, RRISL 221, RRISL 2002 and RRISL 2004) were identified by using the primer OPA 12.

\section{CONCLUSIONS}

Genetic diversity among 35 rubber clones was assessed by using five RAPD primers, namely OPC 04, OPA 18, OPB 18, OPA 20 and OPS 02. The genetic distance coefficient based on RAPD markers ranged between 0.157 and 1.000. The tested 35 clones grouped into seven main clusters at nearly $85 \%$ distance level based on Nei's distant matrix.

Among the primers tested, primer OPB18 and OPS 02 showed the highest polymorphism. The RAPD primer OPC 04, OPA 18, OPB 18, OPA 20, OPS 02 and OPN 18 could be used to identify different clones individually from the set of clone mixtures.

A key was constructed to identify 35 rubb 
er clones using these five primers in RAPD analysis.

\section{REFERENCES}

Annon, (2010). Annual Report, Central Bank of Sri Lanka.

Attanayake, D.P.T.S.G., Herath, S.P., Gamage, A.K., and Karunanayake, E.H., (2000). Identification of RRIC 100 series and two Germplasm clones of Rubber (Hevea brasiliencis) using Random Amplified Polymorphic DNA (RAPD) Technique. Journal of Plantation Crops, 28(2), 163 - 168.

Attanayake, D.P.S.T.G., (2001). Clones for commercial planting in Sri Lanka, Hand book of rubber Vol. 1, Rubber Research Institute of Sri Lanka, pp 46 - 53.

Fonseka, A.A.S., Withanage, S.P. and Attanayake, D.P.T.S.G., (2005). Molecular characterization of recommended rubber (Hevea brasiliensis ) clones of RRISL 200 and RRISL 2000 series by using Random Amplified Polymorphic DNA (RAPD) markers. Proc. of $5^{\text {th }}$ Agricultural Research Symposium 2005. Faculty of Agric. And Plantation Management, Wayamba University of Sri Lanka, pp 41 - 45.

Hermandez, C.H.R., Lucia, A.K., Refael, A.L. and Mario, L.A., (2006). Analysis of genetic variation in clones of rubber (Hevea brasiliensis) from Asian, South and Central American origin using RAPDs marker. Rev. Colomb. Biotecnol.VIII, pp 29 - 34.

Korakot, N., Charassri, N. and Sayan, S., (2008). Analysis of genetic diversity in early introduced clones of rubber tree (Hevea brasiliensis) using RAPD and microsatellite markers Songklanakarin, J. Sci. Technol. 30(5), 553-560.

Low, F.C., Atan, S., Jaafar, H. and Tan, H., (1996). Recent advances in the development of molecular markers for Hevea studies. J. Nat. Rubber Res., 11, 32 - 44.

Nei, M. (1972). Genetic distance between populations, Am. Nat. 106, 283 - 292.

Nei, M. and Li, W.H., (1979). Mathematical model for studying genetic variation in terms of restriction endonuclease., Proc. Nat. Acad. Sci., USA, 76, pp 5269 - 5273.

Rogers, J.S. (1972). Measures of genetic similarity and genetic distance. Stud. Genet. VII, Univ. Texas Publ, 7213, 145 - 153.

Venkatachalam, P., Thomas, S., Priya, P., Thanseem, I., Gireesh, T., Saraswathyamma, C.K. and Thulaseedharan, A., (2002). Identification of DNA polymorphism among clones of Hevea brasiliensis Muell. Arg. using RAPD analysis., Indian J. Nat. Rubber Res., 15, 172181.

Withange, S.P. and Attanayake, D.P.S.T.G., (2005) The simplest method for isolation of Hevea DNA. Bulletin of the Rubber Research Institute of Sri Lanka, 45, pp 36 - 39. 